\title{
Etude comparative des kystes de Naegleria et d'Acanthamoeba (Hartmannella) au microscope électronique à balayage
}

\author{
par CIUREA-VAN SAANEN \\ Institut Universitaire de Microbiologie, Service de Parasitologie, \\ Bugnon 44, 1011 Lausanne, Suisse.
}

\begin{abstract}
RESUME. L'étude faite au microscope électronique à balayage se réfère à la morphologie externe des kystes d'amibes libres. Les amibes du genre Naegleria présentent des kystes dont la surface est dépourvue de plis. Elle peut être lisse ( $N$. fowleri) ou rugueuse $(N$. gruberi). Le nombre moyen de pores varie entre 1,2 et 7,2. Leurs bords peuvent être simples ( $N$. fowleri) ou évaginés $(N$. gruberi). L'épaisseur de la paroi des bords varie entre 0,4 et $1,0 \mu \mathrm{m}$ et le diamètre des pores est d'environ $0,6 \mu \mathrm{m}$. Les kystes de Schizopyrenus, Tetramitus et Didascalus sont plus petits, sans plis et les pores n'ont pas été visibles. Par contre, ceux du genre Acanthamoeba présentent une surface plissée avec des plis peu nombreux et superficiels (A. rhysodes, A. culbertsoni) ou nombreux et profonds ( $A$. castellanii, A. polyphaga). Ces amibes ne présentent pas des pores. L'aspect de la surface des kystes, avec toutes ses particularités est d'un grand intérêt pour la taxonomie des amibes libres du groupe limax.
\end{abstract}

\section{Comparative scanning electron microscopic study of Naegleria and Acantha- moeba (Hartmannella) cysts.}

SUMMARY. The present study was performed with the help of scanning electron microscope and refers to the external morphology of the cysts of the free-living amoebae. The amoebae belonging to the genus Naegleria form cysts whose outer layer shows no wrinkles. It can be smooth ( $\mathrm{N}$. fowleri) or rough (N. gruberi). The average number of pores varies between 1.2 and 7.2. Their margin can be smooth (N. fowleri) or pierced (N. gruberi). The thickness of the bordering cell wall varies between 0.4 and $1.0 \mu \mathrm{m}$ and that of the pore is about $0.6 \mu \mathrm{m}$.

Accepté le 16 mai 1980. 
The cysts of the genera Schizopyrenus, Tetramitus and Didascalus are very small, show no wrinkles in their cell wall and contain no pores. In contrast, cysts in the genera Acanthamoeba show their outer layer superficially plugged with a lesser number of wrinkles (A. rhysodes, A. culbertsoni) or with large or deep wrinkles inside their wall (A. castellanii, A. polyphaga). There are no pores observed in their cysts. Thus the distinguishing morphological features of the cysts have generated important role in the taxonomy of the amoebae of the limax-group.

\section{Introduction}

La microscopie électronique à balayage est de plus en plus employée comme méthode d'étude de la structure externe de différents organismes. L'étude présente se réfère à la morphologie externe des amibes libres sous forme de kystes. La diversité des caractères de leur surface a été déjà utilisée comme critère taxonomique pour définir les genres et les espèces (2).

\section{Matériel et méthodes}

Les souches examinées proviennent de la Collection de l'Institut de Médecine Tropicale d'Anvers (Belgique). Elles ont été isolées à partir des lacs ou des piscines dans différentes régions du monde, ou à partir des produits pathologiques des malades atteints par la méningo-encéphalite amibienne primaire (M.E.A.P.). Les souches d'Acanthamoeba ont été maintenues dans des milieux liquides de Cerva et Willaert (C.G.V. et C.G.V.S.) (7). Les souches de Naegleria ont été maintenues dans un milieu liquide à base d'extrait de levure, peptone et foie concentré (Y.P.L.), avec un supplément nutritionnel de sérum fœtal de veau (1).

Les préparations nécessaires pour cette étude ont été faites au Centre de Microscopie électronique de l'Université de Lausanne, selon la méthode classique qui comporte : une première fixation avec $10 \%$ glutaraldéhide dans du tampon cacodylate

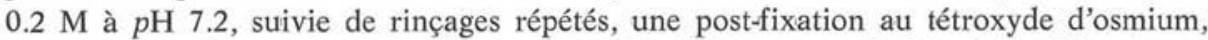
une déshydratation dans des bains successifs d'acétone et d'éthanol, la filtration à l'aide des filtres Nuclépores Shandon, le séchage au point critique et enfin la métallisation à l'or.

Les préparations ont été étudiées au moyen d'un microscope électronique de type JEOL-J.S.M.-35.

\section{Résultats et discussion}

En étudiant comparativement les kystes des Schizopyrenidae et ceux d'Hartmannellidae, nous avons confirmé la différence fondamentale constituée par la présence de replis de la membrane kystique chez les Hartmannellidae, replis absents chez les Schizopyrenidae (totalement absents chez les amibes du genre Naegleria (6). 


\section{A - Etude des kystes appartenant à la famille Schizopyrenidae.}

Les espèces étudiées du genre Naegleria ont été : Naegleria fowleri (aerobia) et Naegleria gruberi. Elles présentent des kystes dont la surface est dépourvue de plis, étant lisse chez $N$. fowleri (fig. 5-7, 12) ou rugueuse chez $N$. gruberi (fig. 1-4). Ces espèces présentent des pores bien visibles préformés dans leur membrane et le nombre moyen varie entre 1,2 chez $N$. fowleri (fig. $6,7,12$ ) et 7,2 chez $N$. gruberi (fig. 1, 2) (3). Les bords des pores sont simples chez $N$. fowleri (fig. 7, 8) ou entourés d'un bourrelet comme une évagination de la paroi, chez $N$. gruberi (fig. 1-4) (5). Les kystes des amibes du genre Schizopyrenus (S. russelli, S. atopus et S. jugosa) sont ronds ou parfois ovales, plus petits et les pores n'ont pas été visibles. Ils sont assez semblables chez ces trois espèces et une différenciation selon leur aspect au balayage serait impossible (fig. 13-16). Les kystes de Didascalus thorntoni et de Tetramitus rostratus se ressemblent aussi : ils n'ont pas de pores, mais leur surface est très finement réticulée (fig. 17, 18).

\section{B - Etude des kystes appartenant à la famille Hartmannellidae.}

Les huit différentes espèces et souches d'amibes du genre Acanthamoeba étudiées, présentent des kystes dont la surface est réticulée avec des plis qui peuvent être peu nombreux et peu profonds, comme chez A. rhysodes (fig. 25), A. polyphaga (fig. 37-40), M. palestinensis (fig. 29, 30), où ils peuvent être nombreux et profonds, comme chez: H. glebae (fig. 35, 36), H. culbertsoni (fig. 31-34), A. castellanii (fig. 4144 ) et $H$. vermiformis (fig. 23, 24). Les pores ont été visibles seulement chez: M. palestinensis (fig. 30), A. rhysodes (fig. 25) et H. astronyxis (fig. 20) : il est possible que toutes ces espèces en possèdent, mais leur visibilité est souvent empêchée par la présence des plis. Etudiant la population kystique chez $A$. rhysodes, on a pu observer la présence de quelques formes transitoires entre le kyste et la forme végétative (fig. 26-28). Ces dernières ont deux types de pseudopodes (acanthopodes) : les uns longs et épais, ayant entre 1 et $2 \mu$ de diamètre, les autres, des filopodes plus courts et plus fins, mesurant $0,1 \mu$ de diamètre (4).

A part la souche Neff d'Acanthamoeba castellanii, on a étudié aussi une autre souche Sn (Snagov) de la même espèce. Ses kystes ont une membrane plissée et une sorte d'ouverture ou fente mesurant $2 \mu$ qui sert au processus de désenkystement, rôle analogue aux pores des autres espèces (fig. 45-48).

A part la présence ou l'absence de plis, à l'aide du balayage il y a d'autres caractères de surface qui peuvent être mis en évidence : la présence des pores ou fentes, leur fréquence et la structure des bords. En conclusion, l'aspect de la surface des kystes offre quelques éléments permettant une différenciation des genres, même si pour une identification précise des espèces d'autres études sont nécessaires.

Les particularités morphologiques des espèces d'amibes libres étudiées se trouvent groupées dans le tableau $I$. 
Tableau I. Etude morphologique comparative des kystes d'amibes libres de type limax.

\begin{tabular}{|c|c|c|c|c|c|}
\hline $\begin{array}{l}\text { Espèce } \\
\text { d'amibe }\end{array}$ & $\begin{array}{l}\text { Aspect de la } \\
\text { surface }\end{array}$ & $\begin{array}{l}\text { Présence des } \\
\text { replis }\end{array}$ & $\begin{array}{l}\text { NB, } \\
\text { moyen } \\
\text { de } \\
\text { pores }\end{array}$ & $\begin{array}{l}\text { Dimen- } \\
\text { sions }\end{array}$ & $\begin{array}{l}\text { Structure } \\
\text { des bords }\end{array}$ \\
\hline $\begin{array}{l}\text { Naegleria } \\
\text { gruberi }\end{array}$ & rugueuse & sans plis & 7.2 & $650 \mathrm{~nm}$. & $\begin{array}{l}\text { entourés } \\
\text { d'un } \\
\text { bourrelet }\end{array}$ \\
\hline $\begin{array}{l}\text { Naegleria } \\
\text { aerobia }\end{array}$ & lisse & sans plis & 1.2 & $800 \mathrm{~nm}$. & $\begin{array}{l}\text { simples, } \\
\text { sans } \\
\text { bords }\end{array}$ \\
\hline $\begin{array}{l}\text { Naegleria } \\
\text { fowleri }\end{array}$ & lisse & sans plis & 1.4 & $800 \mathrm{~nm}$. & $\begin{array}{l}\text { simples, } \\
\text { sans } \\
\text { bords }\end{array}$ \\
\hline $\begin{array}{l}\text { Schizopyrenus } \\
\text { russelli }\end{array}$ & rugueuse & plis petits & - & - & - \\
\hline $\begin{array}{l}\text { Schizopyrenus } \\
\text { jugosa }\end{array}$ & rugueuse & plis petits & - & - & - \\
\hline $\begin{array}{l}\text { Schizopyrenus } \\
\text { atopus }\end{array}$ & rugueuse & plis petits & - & - & - \\
\hline $\begin{array}{l}\text { Didascalus } \\
\text { thorntoni }\end{array}$ & rugueuse & plis petits & - & - & - \\
\hline $\begin{array}{l}\text { Tetramitus } \\
\text { rostratus }\end{array}$ & rugueuse & plis petits & - & - & - \\
\hline $\begin{array}{l}\text { Acanthamoeba } \\
\text { rhysodes }\end{array}$ & lisse & $\begin{array}{l}\text { peu nombreux, } \\
\text { superficiels }\end{array}$ & 0.7 & $600 \mathrm{~nm}$. & $\begin{array}{l}\text { couverts } \\
\text { d'un } \\
\text { bouchon }\end{array}$ \\
\hline $\begin{array}{l}\text { Acanthamoeba } \\
\text { astronyxis }\end{array}$ & lisse & $\begin{array}{l}\text { peu nombreux, } \\
\text { superficiels, }\end{array}$ & 0.5 & $600 \mathrm{~nm}$. & $\begin{array}{l}\text { couverts } \\
\text { d'un } \\
\text { opercule }\end{array}$ \\
\hline $\begin{array}{l}\text { Acanthamoeba } \\
\text { polyphaga }\end{array}$ & lisse & $\begin{array}{l}\text { peu nombreux, } \\
\text { superficiels, }\end{array}$ & - & - & - \\
\hline $\begin{array}{l}\text { Acanthamoeba } \\
\text { castellanii Neff }\end{array}$ & lisse & $\begin{array}{l}\text { nombreux et } \\
\text { profonds }\end{array}$ & - & - & - \\
\hline $\begin{array}{l}\text { Hartmannella } \\
\text { castellanii Sn }\end{array}$ & rugueuse & $\begin{array}{l}\text { nombreux et } \\
\text { petits }\end{array}$ & 1.6 & $2 \mu$ & $\begin{array}{l}\text { ouverture } \\
\text { à bords } \\
\text { simples }\end{array}$ \\
\hline $\begin{array}{l}\text { Acanthamoeba } \\
\text { vermiformis }\end{array}$ & rugueuse & $\begin{array}{l}\text { nombreux, } \\
\text { profonds }\end{array}$ & - & - & - \\
\hline $\begin{array}{l}\text { Mayorella } \\
\text { palestinensis }\end{array}$ & lisse & $\begin{array}{l}\text { peu nombreux et } \\
\text { superficiels }\end{array}$ & 0.6 & $500 \mathrm{~nm}$. & $\begin{array}{l}\text { bords } \\
\text { simples }\end{array}$ \\
\hline $\begin{array}{l}\text { Hartmannella } \\
\text { glebae }\end{array}$ & lisse & $\begin{array}{l}\text { nombreux et } \\
\text { profonds }\end{array}$ & - & - & - \\
\hline $\begin{array}{l}\text { Hartmannella } \\
\text { culbertsoni }\end{array}$ & lisse & $\begin{array}{l}\text { nombreux et } \\
\text { profonds }\end{array}$ & - & - & - \\
\hline
\end{tabular}




\section{Bibliographie}

1. Brent M.M. : Nutritional studies on the Amoebo-flagellate Tetramitus rostratus. Biol. Bull., 1954, 106, 269.

2. Chang S. L.: Small, free-living amebas: cultivation, quantitation, identification, classification, pathogenesis and resistance. Curr. Topics. Comp. Pathobiol., 1971, 1, 201.

3. Jadin J.-B., Eschbach H. L., Verheyen F., Willaert E.: Etude comparative des kystes de Naegleria et d'Acanthamoeba. Ann. Soc. Belge Méd. Trop., 1974, 54, 259.

4. Lastovica A. J.: Scanning electron microscopy of pathogenic and non-pathogenic Naegleria cysts. Intern. J. Parasitol., 1974, 4, 139.

5. Pasternak J. J., Thompson J.E., Schultz T.M.G., Zachariah K. A. : A scanning electron microscopic study of the enkystment of Acanthamoeba castellanii. Exp. Cell. Res., 1970, 60, 290.

6. Schuster F.: An Electron Microscope Study of the Amoeba-flagellate. Naegleria gruberi. 2. The Cyst Stage. J. Protozool., 1963, 10, 313.

7. Willaert E. : Isolement et culture in vitro des amibes du genre Naegleria. Ann. Soc. Belge Méd. Trop. 1971, 51701. 

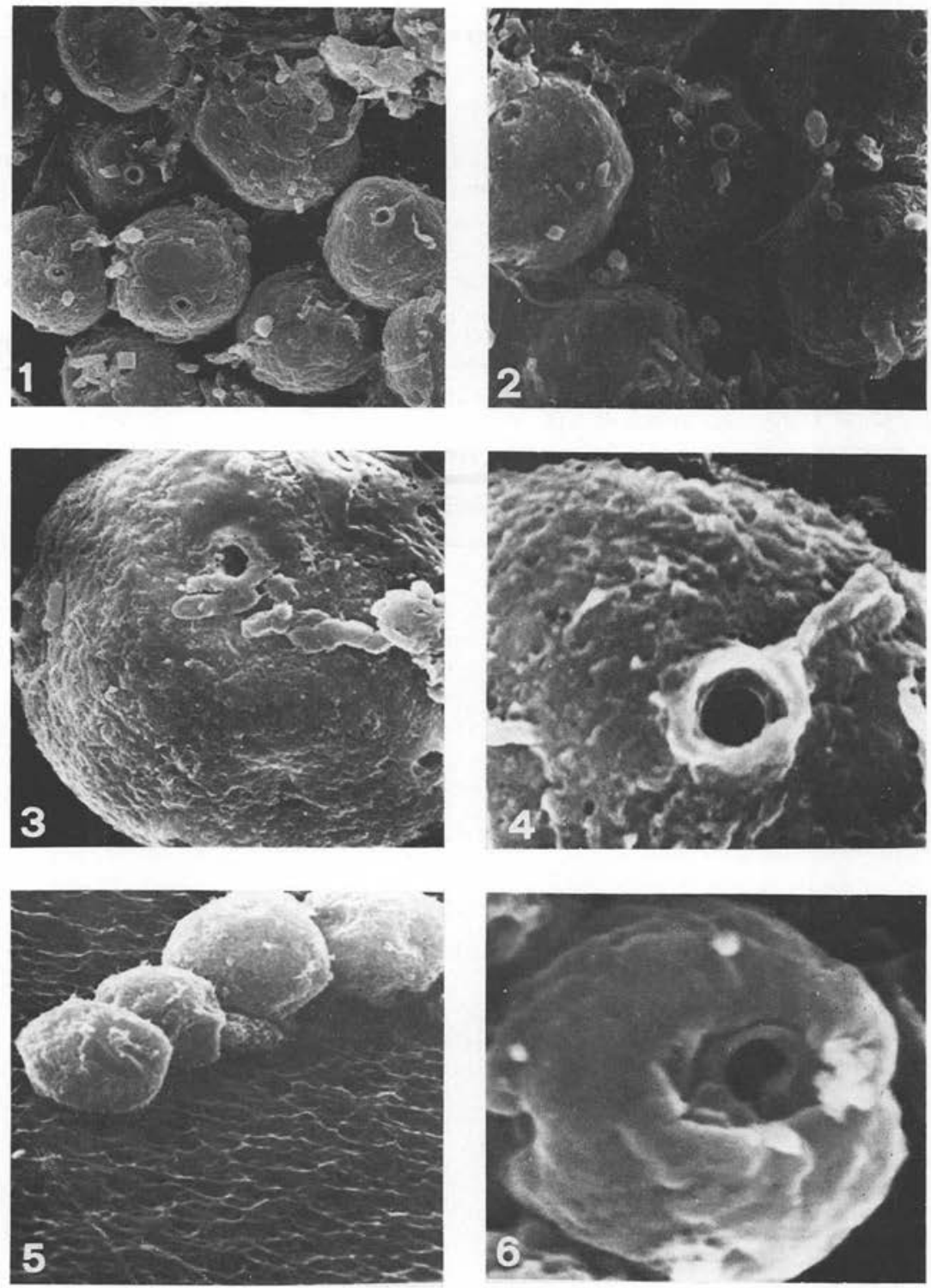

Planche I. - La morphologie externe des kystes d'amibes libres appartenant à la famille des Schizopyrenidae.

Fig. 1 à 4 : Naegleria gruberi; fig. 5 et 6 : Naegleria fowleri. 

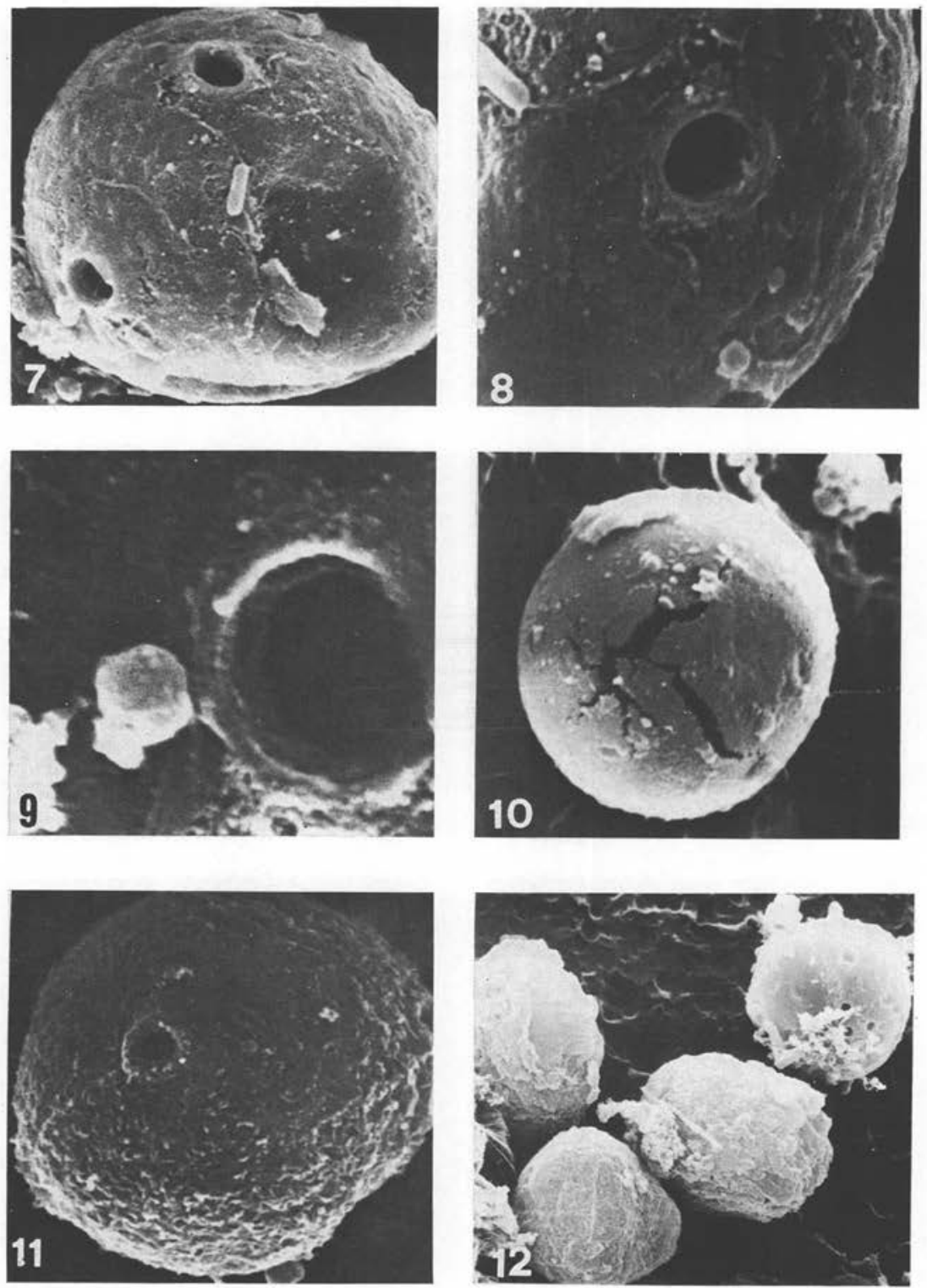

Planche II. - La morphologie externe des kystes d'amibes libres appartenant à la famille des Schizopyrenidae.

Fig. 7 à 12 : Naegleria aerobia. 

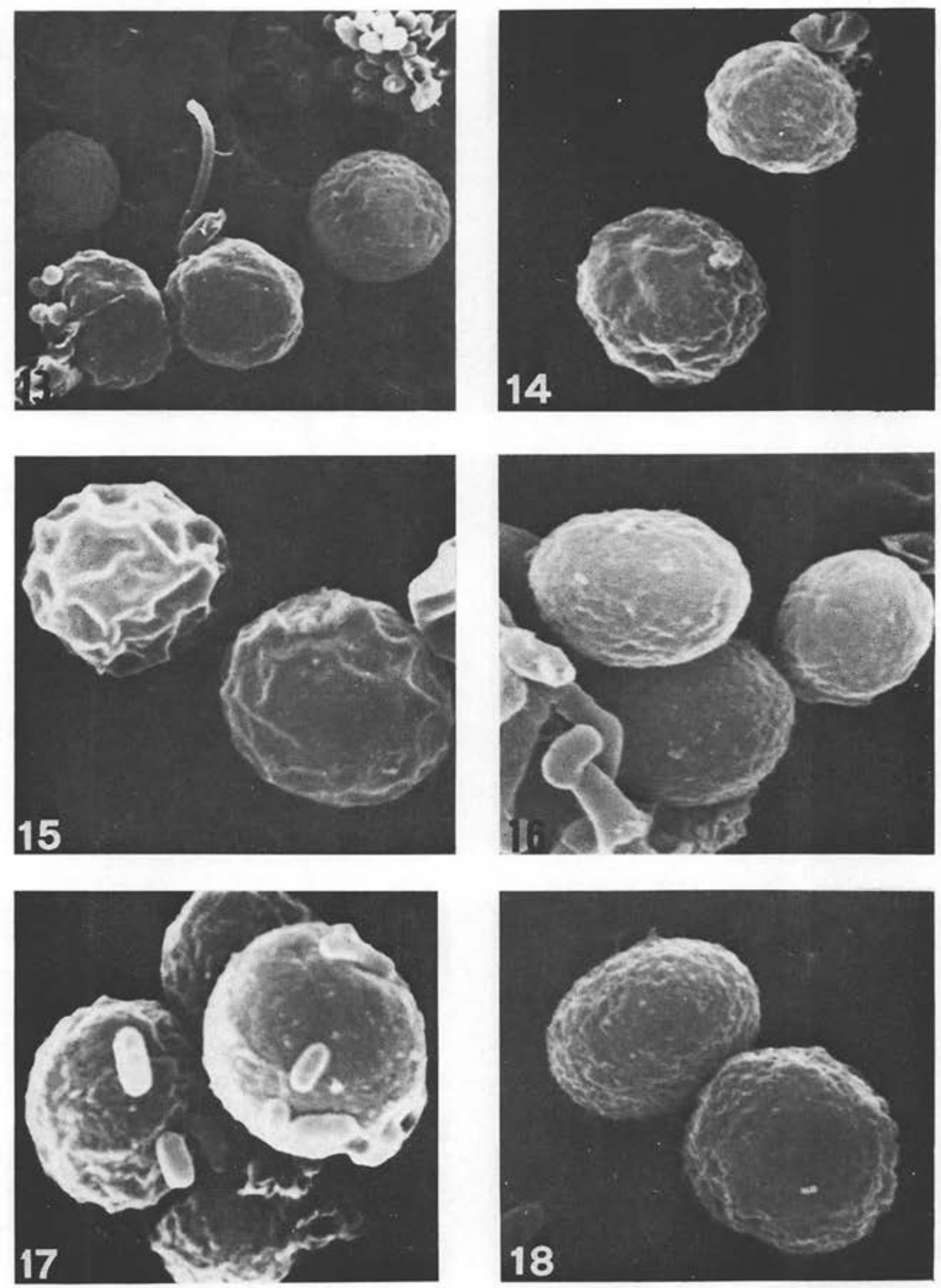

Planche III. - La morphologie externe des kystes d'amibes libres appartenant à la famille des Schizopyrenidae.

Fig. 13 et 14 : Schizopyrenus jugosa ; fig. 15 : Schizopyrenus russelli ; fig. 16 : Schizopyrenus atopus; fig. 17 : Didascalus thorntoni ; fig. 18: Tetramitus rostratus. 

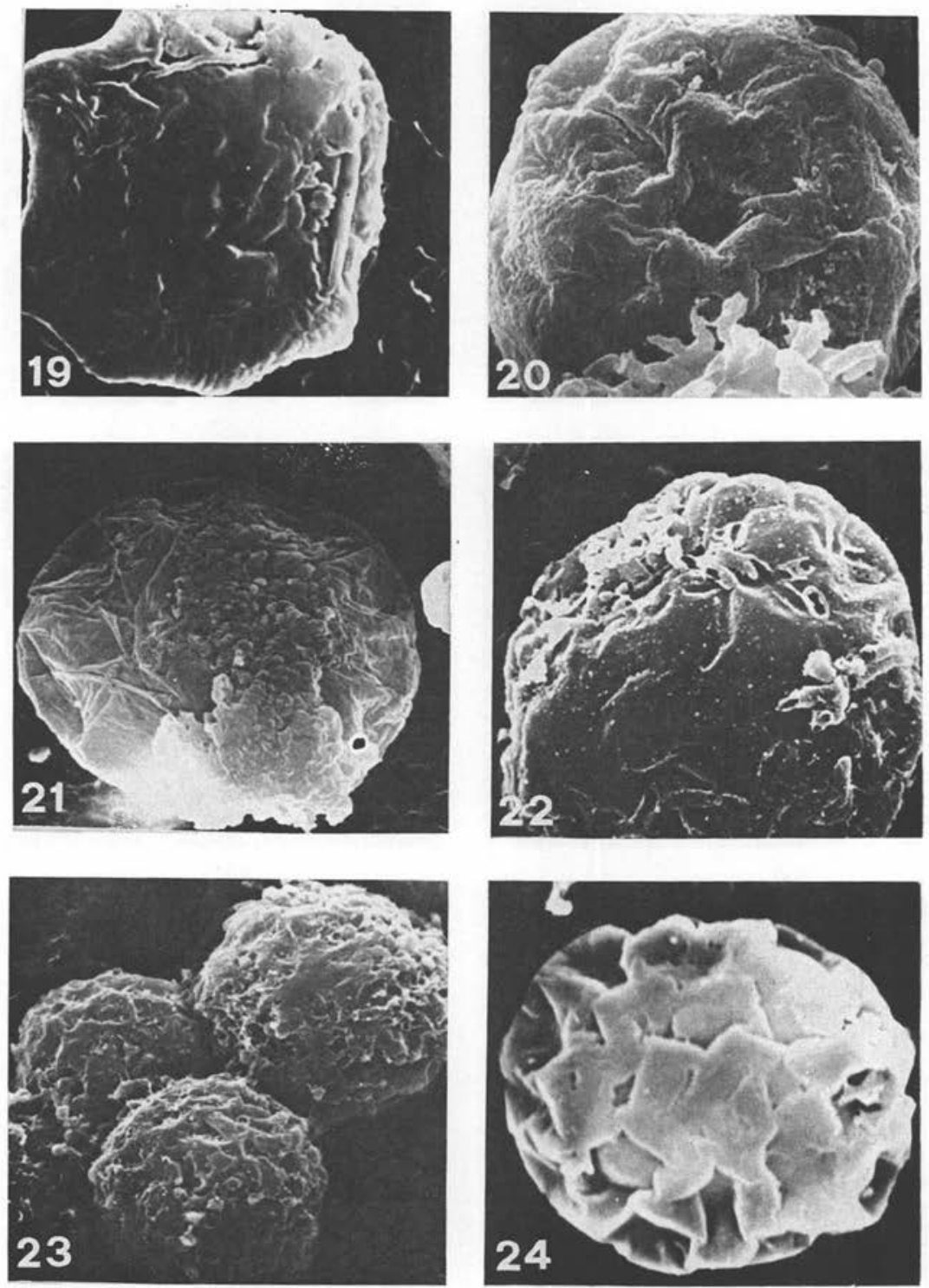

Planche IV. - La morphologie externe des kystes d'amibes libres appartenant à la famille de Hartmamellidae.

Fig. 19 à 22 : Hartmannella astronyxis ; fig. 23 et 24 : Hartmannella vermiformis. 

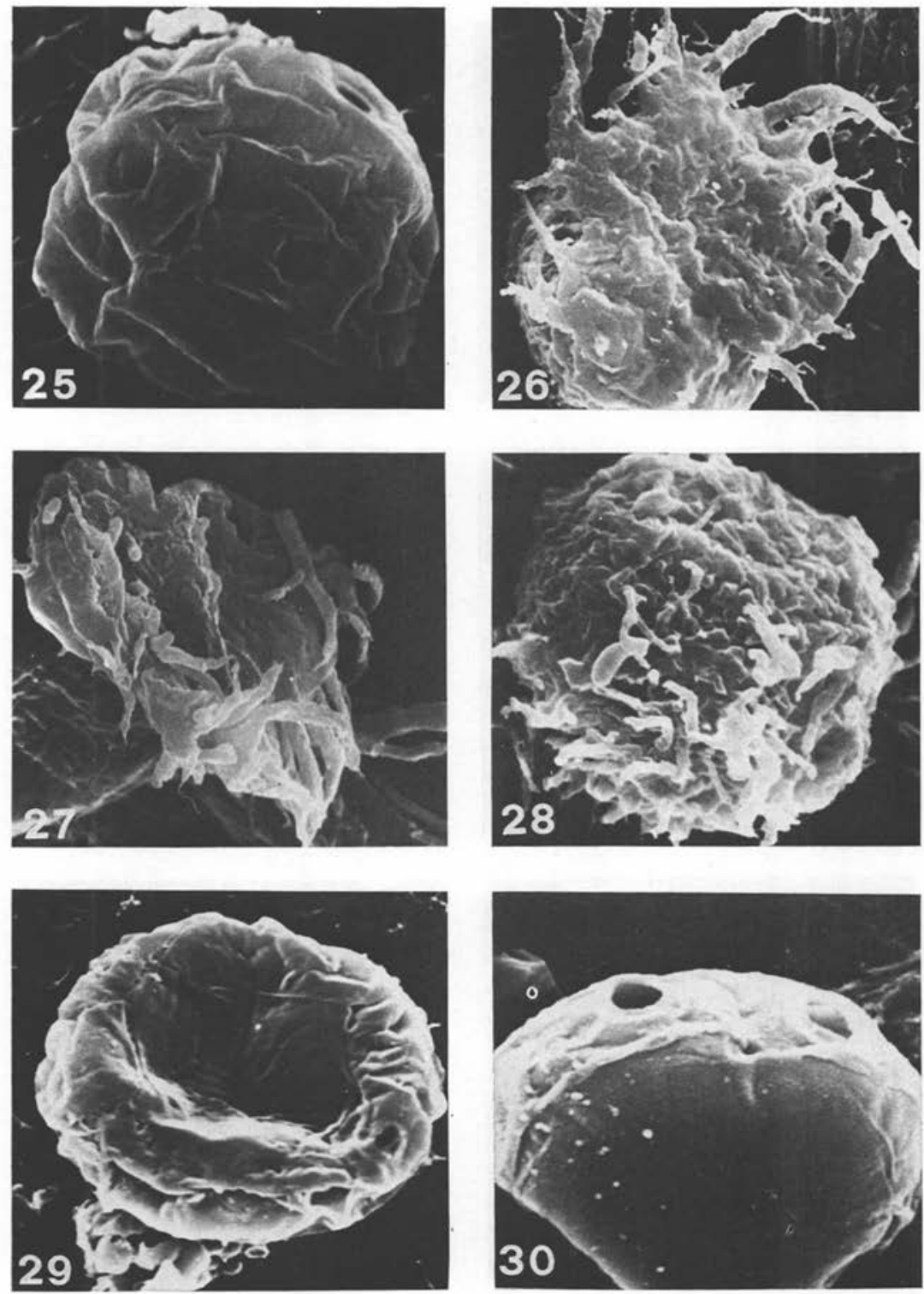

Planche V. - La morphologie externe des kystes d'amibes appartenant à la famille de Hartmannellidae.

Fig. 25 à 28 : Hartmannella rhysodes ; fig. 29 et 30 : Hartmannella palestinensis. 

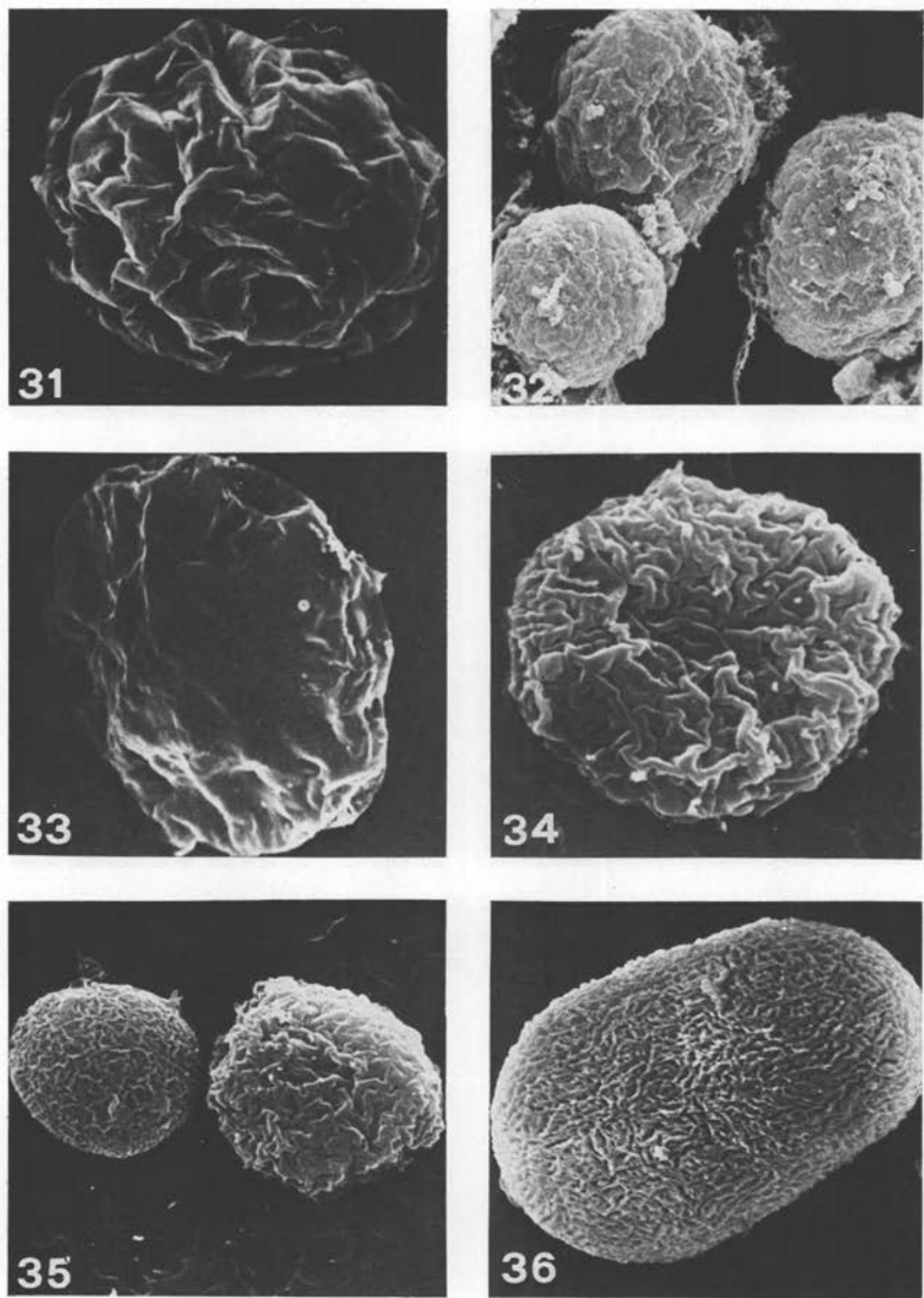

Planche VI. - La morphologie externe des kystes d'amibes appartenant à la famille de Hartmannellidae.

Fig. 31 à 34 : Hartmannella culbertsoni ; fig. 35 et 36 : Hartmannella glebae. 

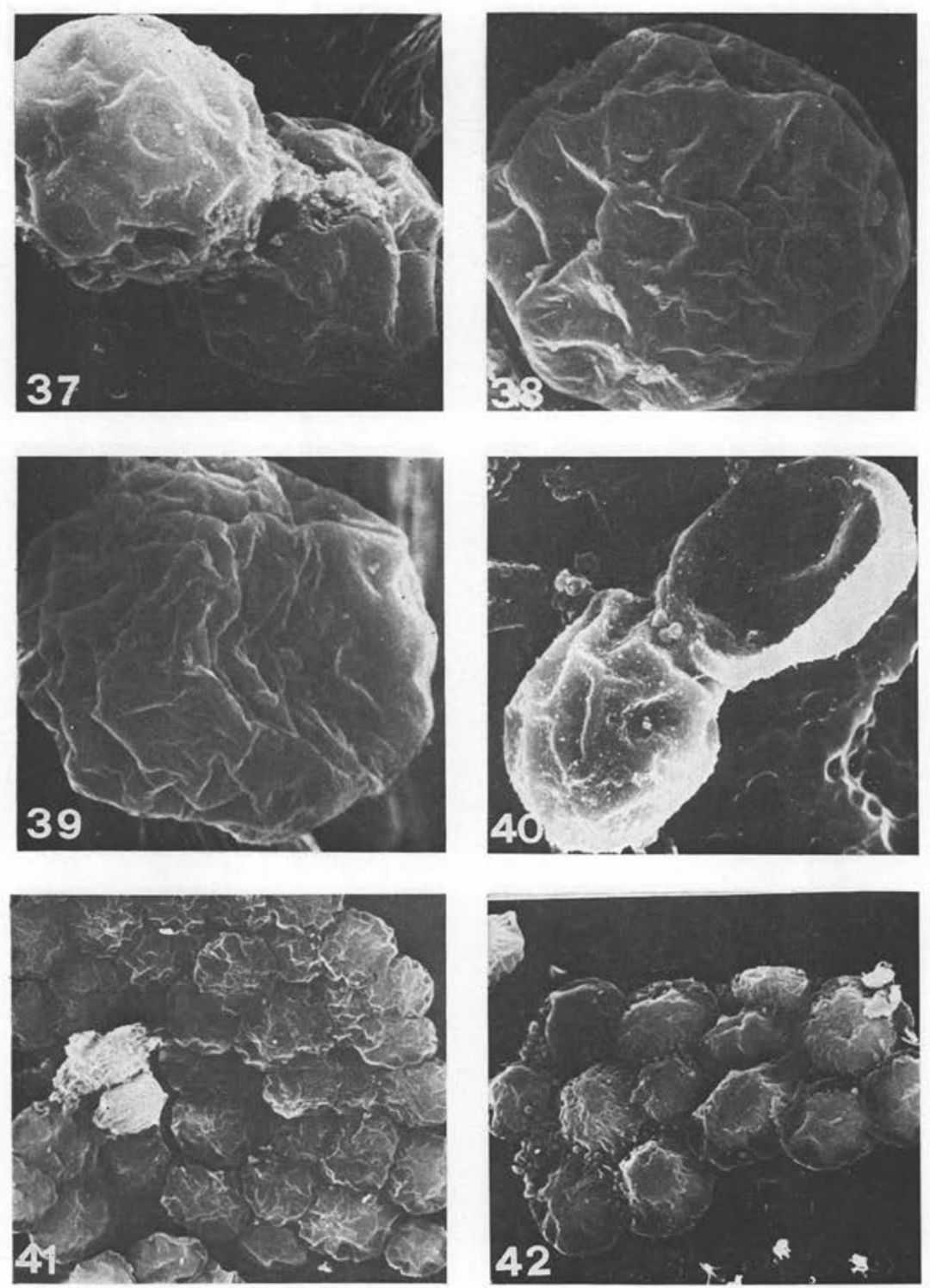

Planche VII. - La morphologie externe des kystes d'amibes appartenant à la famille de Hartmannellidae.

Fig. 37 à 40 : Hartmannella polyphaga ; fig. 41 et 42 : Hartmannella polyphaga. 

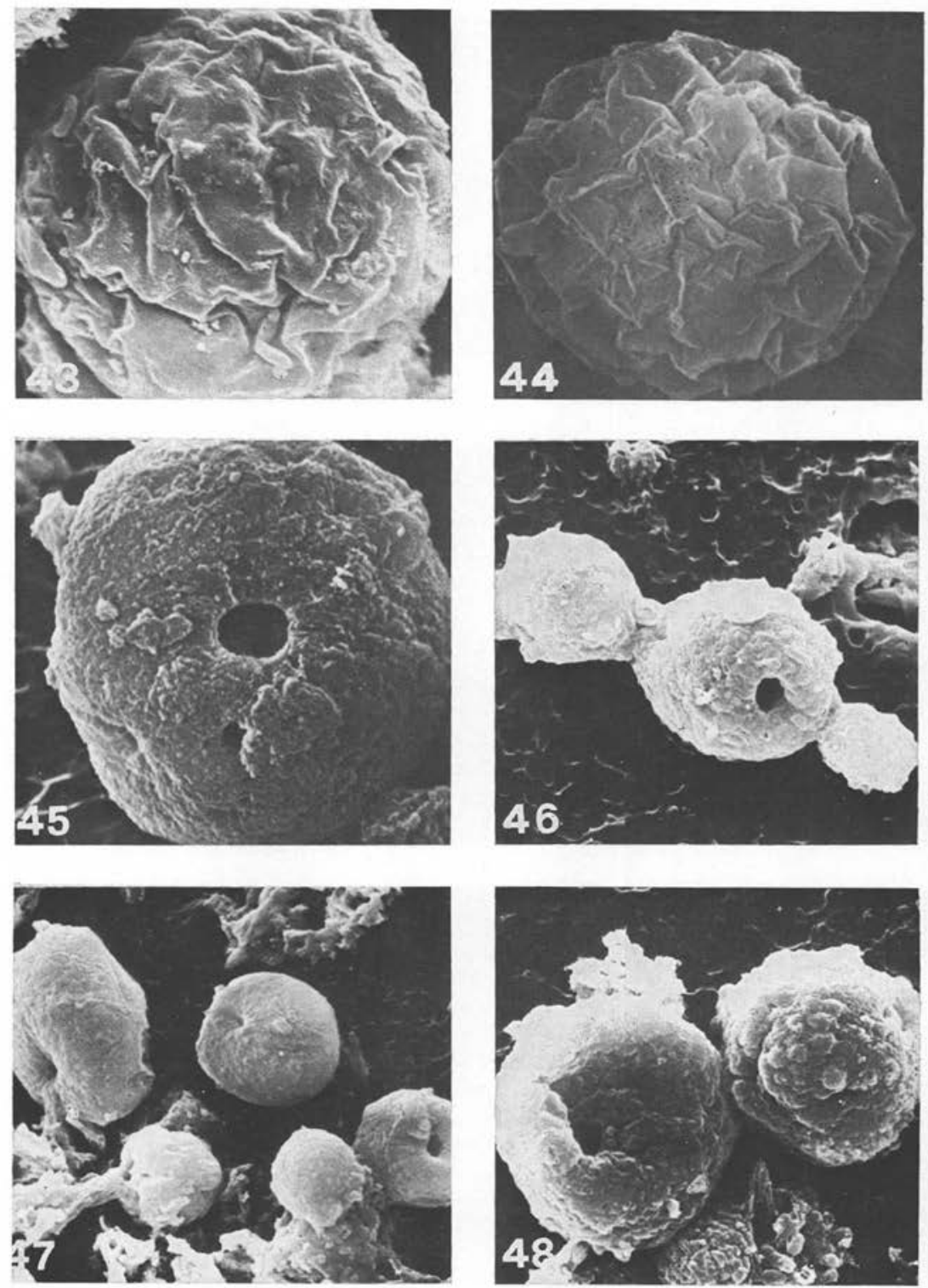

Planche VIII. - La morphologie externe des kystes d'amibes appartenant à la famille de Hartmannellidae.

Fig. 43 et 44 : Hartmannella castellanii (Neff); fig. 45 à 48 : Hartmannella castellanii (Sn). 